\title{
Automatic Recognition and Harvesting of Ripe Tomatoes under Greenhouse Conditions- A Survey
}

\author{
Nivedha Sivakumar ${ }^{1}$, Pushkarini Kulkarni ${ }^{2}$, M. D. Nandeesh ${ }^{3}$ \\ B.E. Student, Electronics and Instrumentation Engg, M. S Ramaiah Institute of Technology, Bangalore, India ${ }^{1,2}$ \\ Assistant Professor, Electronics and Instrumentation Engg, M. S Ramaiah Institute of Technology, Bangalore, India ${ }^{3}$
}

\begin{abstract}
There are numerous challenges in harvesting agricultural products and marketing them effectively. The challenges range from rainfall to availability of seasoned land to labour and marketing supply chain. In manual process, considerable time, hard labour and money is spent in inspecting and harvesting yield. This results in high cost of labour and additional time taken to complete entire process of harvesting, and holistically, deteriorates quality and consistency of the yield. The end-user market is quality demanding and expects consistency within one lot, and also among lots for them to be loyal to the brand and the produce. Often, this is not achievable for reasons discussed above. In many cases, where farm product is input to a final product, say tomato ketchup, high quality and consistency are essential to maintain the quality of the end-product, thereby guaranteeing good customer experience. This paper contains a viable engineering solution to this socially relevant problem of automated harvesting of ripe tomatoes under greenhouse conditions. Image-processing techniques, explained in this paper, are used to identify ripe tomatoes, and once identified; an automatic robotic vehicle harvests the ripe tomatoes.
\end{abstract}

Keywords: Image Processing, Watershed Algorithm, Robotic Harvester, Automated Harvesting, Greenhouse Navigation.

\section{INTRODUCTION}

The objective of this survey is to identify ripe tomatoes on plant stem, under greenhouse conditions, and harvest them by automatic robot. This harvester must be equipped, in terms of hardware and software. It must identify the ripe tomatoes and distinguish them from the non-ripe ones. The basic assumption, in this survey, is that ripe tomatoes can be identified by colour, i.e., redness. This can be backed up by strong positive correlation betweenripeness of tomatoes with the phytochemical responsible for redness of tomatoes,lycopene ${ }^{[1]}$. Raw tomatoes have lycopene content of about $8800 \mathrm{mcg}$, whereas ripe tomatoes contain $2573 \mathrm{mcg}$ of lycopene. Therefore, redness is the most effective factor to detect ripeness of tomatoes.The harvester uses a vision-based system that acquires an image, which is subjected to hyper- spectral analysis to detect ripeness of tomatoes. Algorithm proposed contains four main steps toanalyse the image acquired. Firstly, image background in RGB (Red-Green-Blue) model is removed by removing $\mathrm{G}$ and $\mathrm{B}$ components; thereby, leaving behind only $\mathrm{R}$ component.

Secondly, an algorithm that uses reconstruction operation removes image noise.Thirdly, overlapping tomatoes are to be separated using Watershed algorithm followed by Watershed improvement process. Finally, red tomatoes are detected by merging pixels until they can be merged no more.Ripe tomatoes are identified and the harvesting process can be initiated. Automatic harvesting robot is to be programmed for navigation and task execution. Infrared sensors can achieve obstacle avoidance. It is also provided with task specific end-effectors to pick and place tomatoes. The end-effectors can either have a parallel-plate configuration or a three-finger gripper, with suction in between to grip the tomato and place it in a utility area on the robot.

\section{MATERIALS ANDMETHODS}

Tomatoes having more than $90 \%$ redness are considered ripe, under greenhouse conditions without artificial lighting. All other tomatoes, i.e., the pink, yellow and green tomatoes are considered non-ripe; therefore, these are not harvested. This implementation would be for identifying ripe tomatoes spontaneously; however, some time buffer is necessary between harvesting and marketing to ensure optimal ripeness at the time of usage. In this case, the level of redness can be reduced from $90 \%$ to a different desired value. Although estimated accuracy for this system, with natural lighting, is approximately $80 \%$, it can be improved upon by incorporating artificial lights for image acquisition. A Charge Coupled Device camera with high resolution is to be employed for image acquisition.

\section{A. Identification of Red Tomatoes}

A four-step approach is followed to process the acquired image to distinguish ripe tomatoes from the rest.

1) Remove Image Background:It is necessary to remove image background to detect red tomatoes to remove all green leaves, brown twigs and other peripheral objects from the image. Converting the acquired image into its corresponding RGB model can do this.Now, blue and green components are subtracted from the model obtained to leave behind only red components. The result of this subtraction is a binary image with black background. Binary AND operation is used to separately multiple R, G and B components to remove background. An example of the RGB model of a colour image of ripe tomatoes is as shown in Fig. 1.

2) Noise Removal: Cultivation of tomatoes in parallel rows can be observed in a conventional greenhouse environment. Next step in image processing is to remove noisy tomatoes from parallel rows, i.e., undesired rows. 


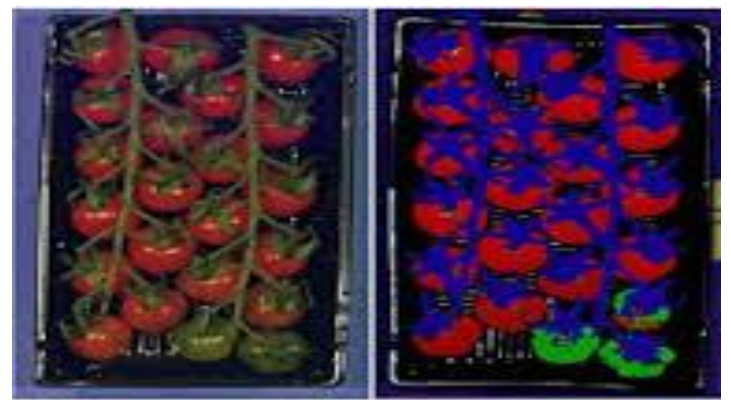

Fig1.RGB model of a colour image of ripe tomatoes

These tomatoes on undesired rows can be distinguished from desired tomatoes from distance of tomatoes from the point of acquisition of image. This can be deduced from comparison of tomato size with a certain threshold, as tomatoes farther away are smaller. Opening algorithm with reconstruction operation is used to meet this end. Reconstruction operation can be understood as a conditional dilation, where pixels of image are dilated only if corresponding pixel in the mask exists.

3) Separating Tomato Clusters: It can be observed that tomatoes often grow in clumps, but can only be harvested one at a time. Therefore, it is essential to reduce these clumps of tomatoes to individual units, and harvest them individually using image segmentation. An effective method used for this application is Watershed algorithm, which interprets the image as a height profile, which is flooded starting at regional minima. Watershed algorithm is especially suited for circular objects, as is the case here. Since Watershed algorithm, by itself, is known to give inaccurate results at times due to over-segmentation, we use it in company of Watershed Improvement Process (WIP). The Watershed Improvement Process is executed in steps as follows:

- Two-dimensional Euclidean distance transform of the binary image is calculated.

- Counting objects in image from previous step calculate the number of objects in the image.

- Erosion operation is performed until all overlapping tomatoes are separated.

- Over-segmented components are merged.

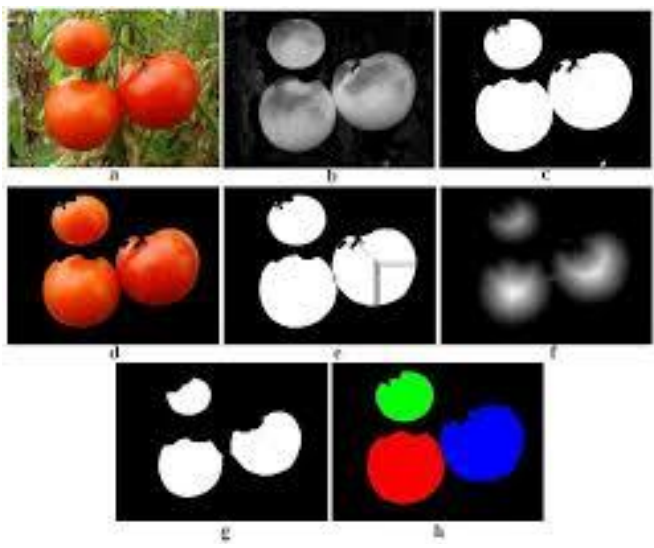

Fig.2. Image Processing operations before recognition of red from non-red tomatoes (a) Original colour image (b)Grey scale image (c) Binary image (d)Image after removing background (e)Separated objects by Watershed Algorithm (f)The 2-D Euclidean distance transforms $(\mathrm{g})$ Erosion operation (h)Joining of over segmented objects.

4) Red Tomato Detection: Region growing approach, an image segmentation technique, is utilised to detect red tomatoes. Here, pixels with a high degree of correlation are grouped together to form a segmented region until no more pixels can be merged. One needs to start with 'seed points', which can be selected based on image type. For monochrome images, region analysis must be carried out with descriptors based on grey levels or textural properties. Now colour space transformation is carried out, where the resultant colour image from previous step is converted into a HSI (Hue-Saturation-Intensity) model.This step is necessary as HSI model is essential to differentiate between colours, and also shades of the same colour. A non-linear relation from RGB, using the following equations, calculates HSI.

$$
\begin{gathered}
\mathbf{H}=\arctan \frac{\sqrt{3}(\mathbf{G}-\mathbf{B})}{(\mathbf{R}-\mathbf{G})+(\mathbf{R}-\mathbf{B})}(\mathbf{1}) \\
\mathbf{S}=\mathbf{1}-\frac{\mathbf{3}}{(\mathbf{R}+\mathbf{G}+\mathbf{B})} \min (\mathbf{R}, \mathbf{G}, \mathbf{B})(\mathbf{2}) \\
\mathbf{I}=\frac{\mathbf{1}}{\mathbf{3}}(\mathbf{R}+\mathbf{G}+\mathbf{B})
\end{gathered}
$$

Applying the above equations, HSI model for any RGB model can be obtained. Hue $(\mathrm{H})$ is the differentiator, as it represents basic colour. Region analysis is carried out from hue information.Basic region growing can be achieved on MATLAB software using the below syntax:

$$
\text { [Output]=region grow }(\mathrm{F}, \mathrm{S}, \mathrm{T})
$$

where $\mathrm{F}$ is an image to be segmented and parameter $\mathrm{S}$ can be an array or a scalar. If $\mathrm{S}$ is a scalar, it defines an intensity value such that all the points in $\mathrm{F}$ with that value become seed points. Similarly, T can be an array or a scalar. If $\mathrm{T}$ is a scalar, it defines a global threshold. The threshold value is used to test if a pixel in the image is sufficiently similar to the seed. If the absolute value of the difference between the seed and its neighbouring pixel is less than or equal to $\mathrm{T}$ that pixel joins to seed, but not otherwise.

\section{B. Robotic Subsystem}

Robot is used for plant inspection, harvesting of fruits and automatic navigation. Key to deciding the type of robotic harvester and its end-effector are the operating conditions in greenhouse. Greenhouses are generally translucent buildings for rearing or hastening the growth of plants. This agricultural technique is very popular for horticultural production in regions with harsh climatic conditions. The distribution of plants inside greenhouses for plant growth is usually double row, with narrow corridors for human operation. Robot must traverse these narrow columns to harvest ripe tomatoes. The harvester navigates autonomously along greenhouse corridors by means of a sensor system that eliminates human intervention. The requirements to be considered for development of a prototype are reliability, navigation in unaltered greenhouses, low cost, flexibility, autonomous operation and robust control system for safe operation. The performance of robotic manipulator is completed by the 
end effector with three degrees of freedom, which carries out pick-and-place operation on the ripe tomatoes, identified by image processing techniques described above.

C. Greenhouse Navigation and Operation

To maximize the number of plants grown within a greenhouse, the traversal space is designed to be narrow. It is important for the robot to navigate without altering its environment. It can be done by one of two approaches: planned and reactive. Planned method relies on a map that models the geography, with position estimates. Reactive method is based on object perception and plans its route by finding a safe path, by avoiding obstacles.For this application, using the reactive approach would ensure robots reaching their target positions, and rules out collision with obstacles.

\section{CONCLUSION}

Results with good accuracy can be expected in areas with sufficient and uniform lighting, as the images obtained are of good accuracy. The usage of HSI model could considerably reduce the effect of uncontrolled lighting.In background removal; good segmentation is achieved when high contrast exists between object and background. Care should be taken to ensure that red coloured objects, other than tomatoes, are not in the robot's line-of-sight to prevent discrepancies in results. Separationof tomato clusters uses Watershed algorithm with improvement process to achieve good accuracy.Here, merging oversegmented componentsand counting the number of objects in the imageusing the improving process can compensate possible over-segmentation of image.Erosion is performed to segregate over-lapping tomatoes to obtain defined boundaries.

\section{REFERENCES}

[1] T. Burks, F. Villegas, M. Hannan, B. Sivaraman, V. Subramanian, S. Flood and J. Sikes, "Engineering and Horticultural Aspects of Robotic Fruit Harvesting: Opportunities and constraints," HorTechnology, vol. 20, pp. 569-571, Nov. 1999.

[2] Alireza Khoshroo, Arman Arefi and Jalal Khodaei, "Detection of Red Tomato on Plants using Image Processing Techniques," Agricultural Communications, 2(4): 9-15, Sep. 2014.

[3] Rafael C. Gonzales, Richard Woods, Digital Image Processing, $3^{\text {rd }}$ ed., 2009.

[4] Jos B.T.M. Roerdink and Arnold Meijster, "The Watershed Transform: Definitions, Algorithms and Parallelization Strategies," Fundamenta Informaticae, 2001

[5] Hosna Mohamadi, Mahmoud Omid, R. Alimardani "Detection of red ripe tomatoes on stem using Image Processing Techniques", Journal of American Science, May 2011.

[6] Naoshi Kondo "Fruit Harvesting Robot and Grading Robot" inJoint Conference on Environmental Engineering in Agriculture, 2009.

[7] Anurag Sharma, "Design Study of End Effectors", International Journal of Engineering and Advanced Technology (IJEAT), ISSN: 2249 - 8958, Volume-4 Issue-3, Feb. 2015.

[8] Anthony Mandow, Jesus M Gomez, Jorge Luis Martinez Rodrigues, Victor F. MuAoz, Anibal Ollero, Alfonso Garcia-Cerezo, "The Autonomous Mobile Robot AURORA for Greenhouse Operation" IEEE Automation and Robotics Magazine, Dec. 1996.

[9] Simon Blackmore, Bill Stout, Maohua Wang, Boris Runov "Robotic agriculture- The future of agricultural mechanization" in 5th European Conference on Precision Agriculture Uppsala, Sweden, 9-12th June 2005. 